\title{
Measurement of Spontaneous Undulator Radiation Statistics Generated by a Single Electron (URSSE)
}

\author{
Ihar Lobach \\ University of Chicago \\ Sergei Nagaitsev \\ Fermi National Accelerator Laboratory and \\ University of Chicago \\ Giulio Stancari, Aleksandr Romanov, and Alexander Valishev \\ Fermi National Accelerator Laboratory \\ Aliaksei Halavanau and Zhirong Huang \\ SLAC National Accelerator Laboratory
}

(Dated: September 2, 2020)

\section{MOTIVATION}

As previously stated in the experiment proposal [1], the goal of the URSSE experiment was to study statistical properties of the undulator radiation generated by a single electron, circulating in the IOTA storage ring. Namely, the idea was to study the distribution of the number of photons detected in a certain time window, and the distribution of the inter-arrival times of the photons. We wanted to see if the photostatistics was exactly Poissonian, as predicted by [2-4], or if there was some deviation towards Sub-Poissonian light, similar to what was observed in [5].

There were 4 URSSE shifts:

- Wed 2020-02-26

- Fri 2020-02-28

- Tue 2020-03-03

- Thu 2020-03-05 


\section{APPARATUS}

A diagram of the final version of the apparatus that was used in the experiment is shown in Fig. 1. We used the Single Photon Avalance Diode to study the undulator radiation generated by a

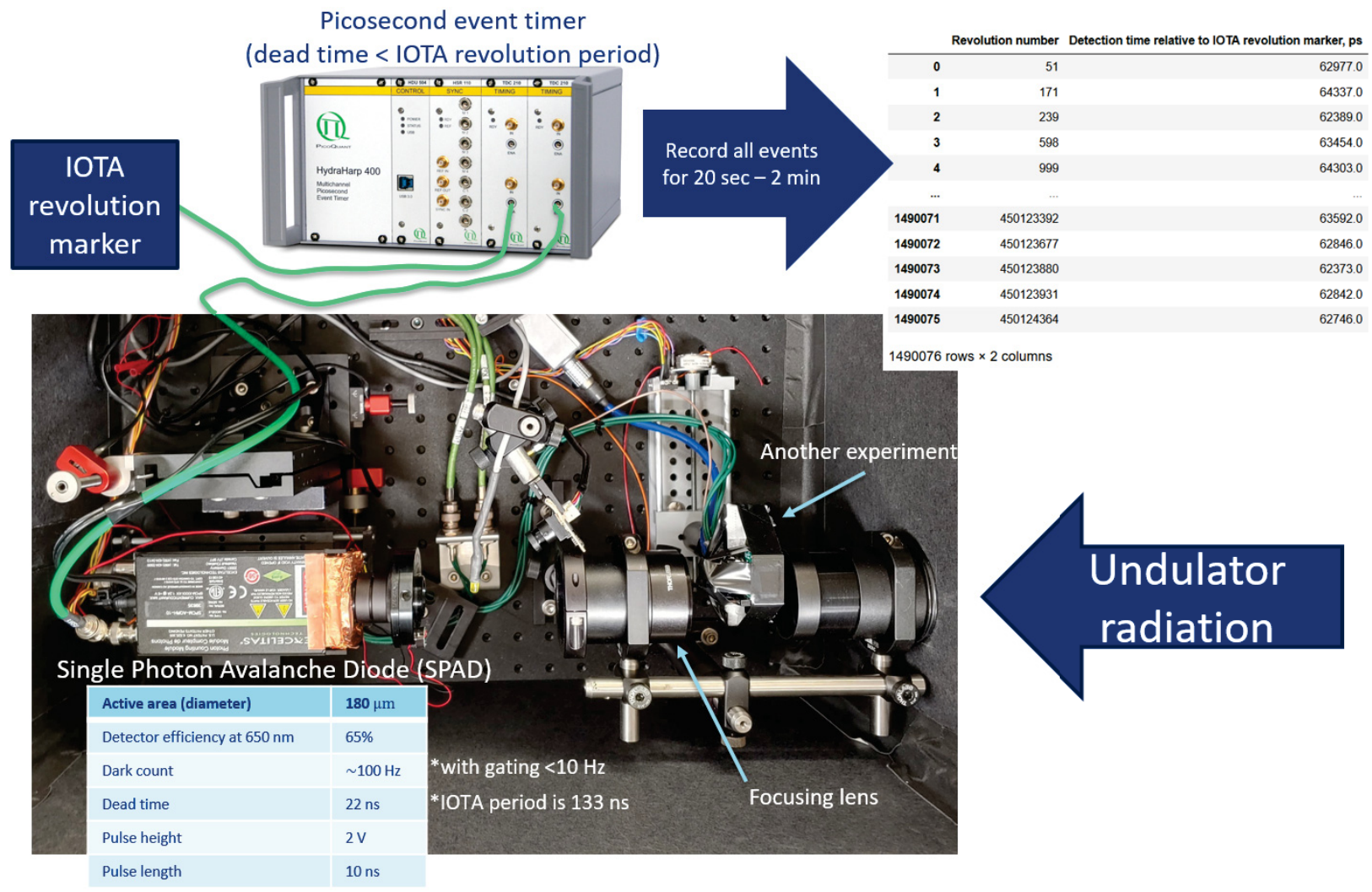

FIG. 1. Diagram of the apparatus.

single electron. Some properties of this detector are provided in Fig. 1, a more detailed description is provided in [1], also please see the experiment's website [6]. The undulator radiation was focused on the sensitive area of the SPAD detector by a single focusing lens. The pulses from the SPAD detector went to the picosecond event timer HydraHarp 400, which was also provided with the IOTA revolution marker. In this way, we were able to obtain a table containing a revolution number and a detection time relative to IOTA revolution marker for each detection event, see Fig. 1.

The SPAD detector was mounted on a 3D movable stage. For the motion in transverse direction $(x$ and $y)$ we used picomotors. For the longitudinal direction $(z$ - along the radiation) we used a translation stage with a stepper motor. 


\section{ALIGNMENT AND VERIFICATION OF SPECTRAL-ANGULAR PROPERTIES OF THE RADIATION}

To find the optimal location of the detector we moved it iteratively along the $x, y$, and $z$ directions. An example of such scan along the $x$-direction in the vicinity of the optimal location is shown in Fig. 2.

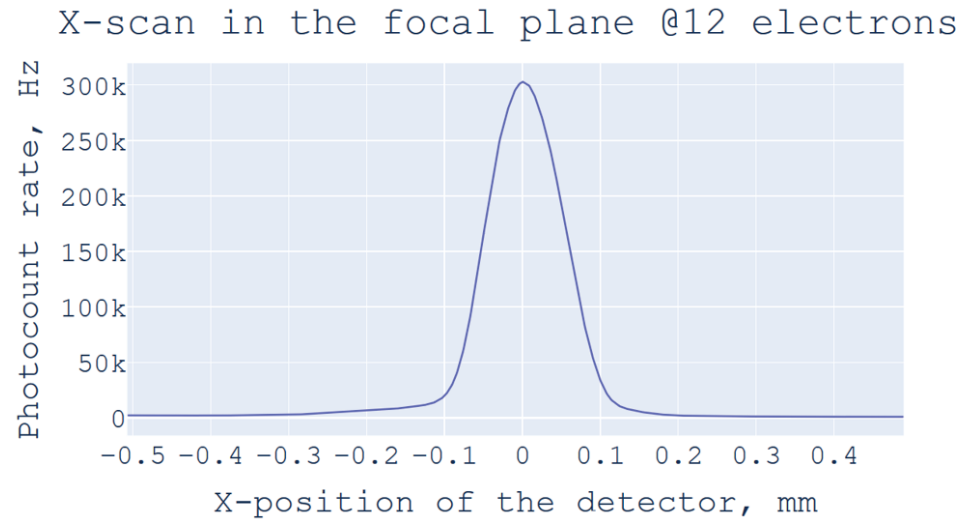

FIG. 2. Photocount rate as a function of $x$-position of the SPAD detector in the vicinity of the optimal location.

The measured photocount rate in the optimal location was about $25 \mathrm{kHz}$ per electron, or about one photocount per 300 IOTA revolutions, on average. The value of the photocount obtained in a simulation using the Synchrotron Radiation Workshop (SRW) package [7] is $53 \mathrm{kHz}$.

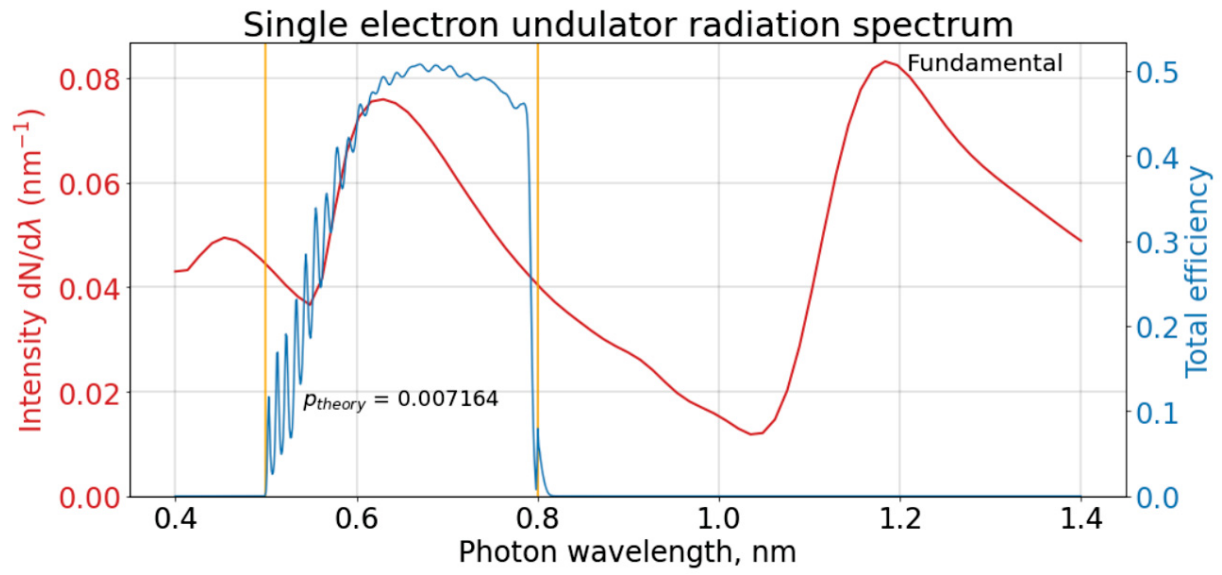

FIG. 3. The undulator radiation spectrum, integrated over the lens area.

The simulation took into account the transmission functions of the two mirrors, the vacuum chamber window, the lens, the low-pass $(500 \mathrm{~nm})$ and high-pass $(800 \mathrm{~nm})$ filters, and the quantum 
efficiency of the SPAD detector. However, it dit not take into account the possible aberrations in the lens, which probably resulted in a rather significant light spot size in the optimal location of the detector. This light spot size was comparable with the size of the sensitive area of the detector, which was about $180 \mu \mathrm{m}$. Therefore, this is a likely explanation for the fact that about a half of the photons were lost. Nonetheless, $25 \mathrm{kHz}$ is much larger than the dark counts rate, which was only about $10 \mathrm{~Hz}(100 \mathrm{~Hz}$ without gating).

We also decided to compare the simulated and the measured angular intensity distributions, see Fig. 4.
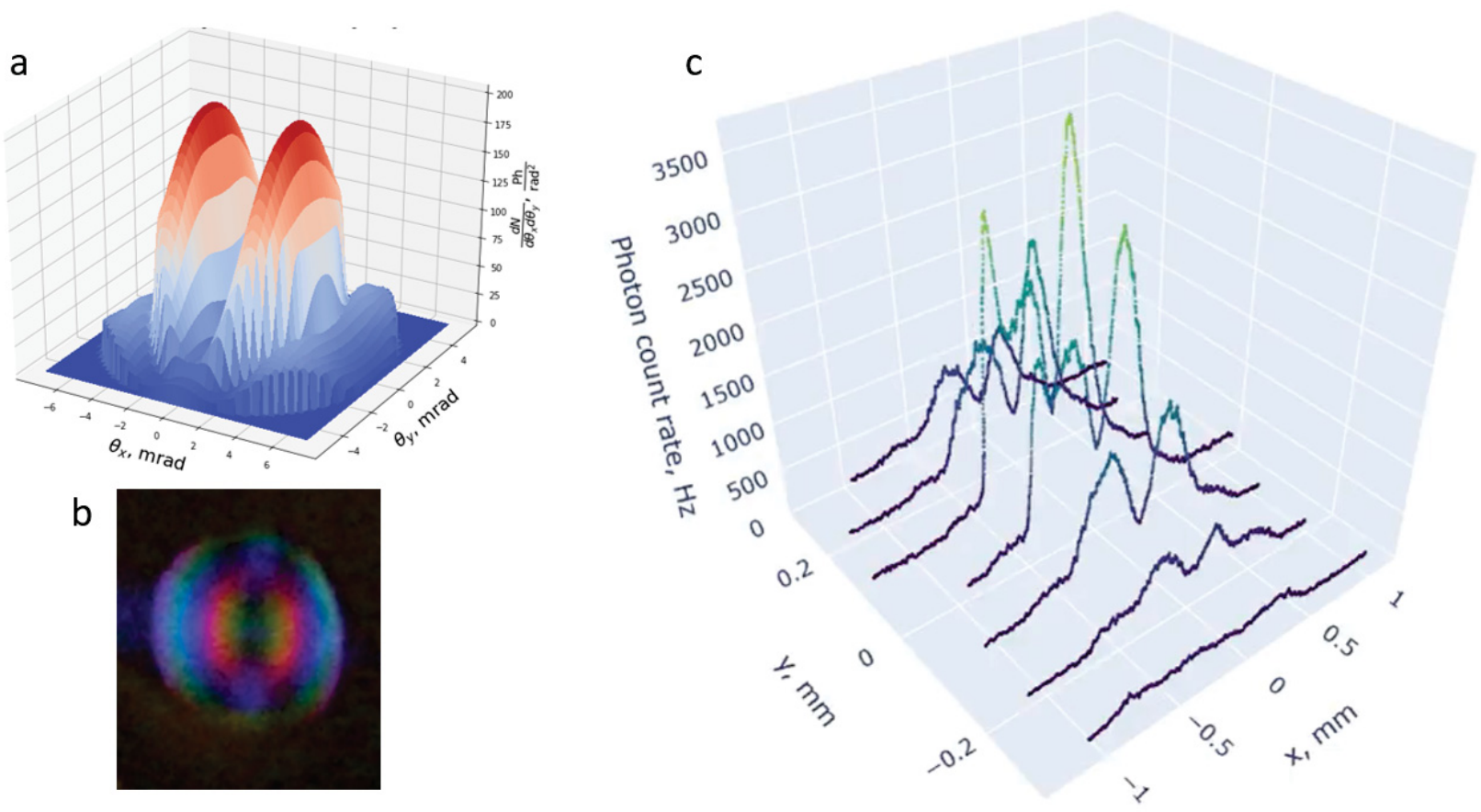

FIG. 4. (a) SRW simulation for the angular distribution of the number of detected photons. (b) Photograph of the undulator radiation on the surface of the optical shutter. (c) Seven horizontal scans of the SPAD photocount rate at different vertical positions of the detector.

In Fig. 4 a, a simulation with the SRW package is presented. Since we are working with the second harmonic of undulator radiation, the intensity on axis goes to zero, and there are two peaks on the sides. The photograph of the undulator radiation on the surface of the optical shutter in IOTA shown in Fig. 4 b looks very similar to the simulation, shown in Fig. 4 a. We also attempted to study the angular intensity distribution with our SPAD detector, see Fig. 4 c. We moved the detector left and right at seven different vertical positions to see how the photocount rate changes. This had to be done far away from the optimal locations of the detector (focal plane), because in the focal plane we would only see on small light spot and we wouldn't be able to observe any angular 
structure of the radiation. In Fig $4 \mathrm{c}$, the coordinate $x$ is the time of travel of the picomotor, converted into distance using the picomotor's specifications. The $y$ coordinate, is the number of steps, converted into distance, using the specifications of the picomotor. It was an open-loop picomotor, therefore, it should be understood that Fig. 4 c depicts the angular distribution very approximately.

\section{STUDY OF THE PHOTON STATISTICS}

The collected data can be represented as a series of zeroes and ones,

000001000000000000100000001100000000000001000000001110000,

where every zero represents IOTA revolution without a detection event and every one represents IOTA revolution with a detection event. In analogy with the energy-loss fluctuations of a charged particle in matter, photon statistics can be characterized by the Fano factor,

$$
F=\frac{\operatorname{var}(\mathcal{N})}{\langle\mathcal{N}\rangle}
$$

where $\mathcal{N}$ is a number of photons detected in a certain time window. This time window can be a single IOTA revolution, or a certain number of IOTA revolutions. Depending on the value of the Fano factor the photostatistics can be

- $F=1-$ Poissonian

- $F>1$ - Super-Poissonian

- $F<1$ - Sub-Poissonian

Poissonian photostatistics is very common, e.g., laser radiation, radioactive decay, etc. SuperPoissonian is perhaps less known, but also very common. There are such examples as thermal radiation, any classical fluctuations of radiation intensity, fluctuations of incoherent synchrotron radiation generated by an electron bunch [8-12]. Sub-Poissonian photostatistics is unusual and indicates some interesting quantum state of the radiation. Therefore, it would be unexpected to see this kind of photostatistics in synchrotron radiation which typically can be fully described classically (except for quantum excitation, of course). However, sub-Poissonian light was observed 
in synchrotron radiation before at least once, namely in the seventh harmonic of FEL radiation, as reported in $[5,13]$. This is why we wanted to carry out a precision study of photostatistics of undulator radiation in IOTA to see if there is any deviation from classically predicted Poissonian photostatistics.

Figure 5 shows the results of the analysis of one 60 -second-long data set for a single electron, circulating in IOTA. The number of detection events in this data set is 1484118 . One could calculate the Fano factor for the entire 60-second-long data set, but this value would not be useful because we would not know the confidence interval for this value. Therefore, we divided the 60second-long data set into 1000 sub-samples and calculated the Fano factor in each of them. Then, we made a histogram of the 1000 values, see Fig. 5.

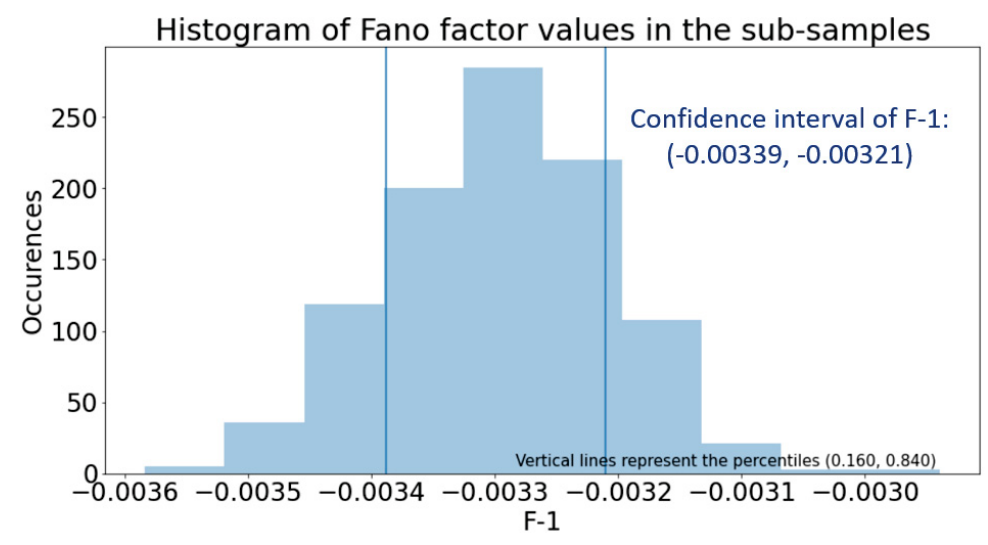

FIG. 5. Histogram of 1000 values of the Fano factor $F$, corresponding to 1000 sub-samples of one 60 second-long data set.

The histogram in Fig. 5 shows that all 1000 values of $F$ are slightly less than one. At first sight, this is a very interesting and unexpected result - sub-Poissonian photostatistics. However, after a more detailed consideration we understood that this slight deviation of $F$ from one is perhaps related to the principal of operation of the SPAD detector. Namely, our SPAD detector is binary, i.e., it can only produce zeros and ones, see (1). After each detection the detector creates a pulse of the same width and height, see Fig. 6.

If our detector could see the difference between detections of $1,2,3, \ldots$ photons, then some of the ones in (1) would be replaced by $2,3, \ldots$

$$
000001000000000000200000001100000000000001000000001310000 .
$$

Therefore, we believe that during our measurements the Poisson distribution of the number of 


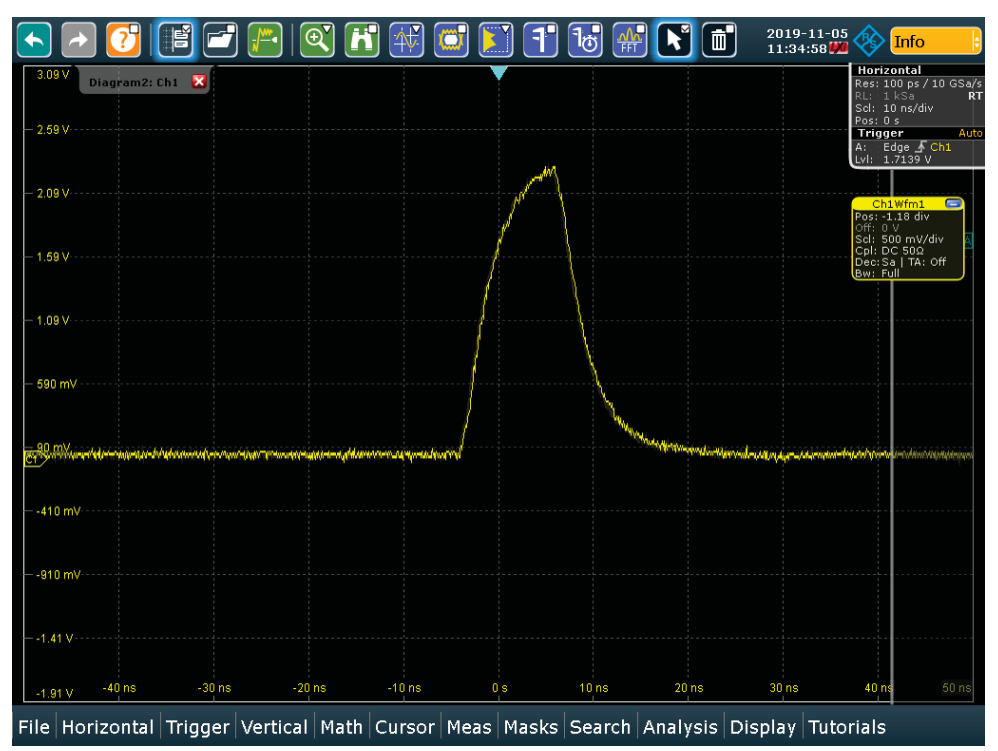

FIG. 6. Waveform of a SPAD pulse corresponding to a photon detection event.

photons,

$$
\operatorname{Pr}(k)=\frac{\lambda^{k}}{k !} e^{-\lambda}
$$

was converted into the Bernoulli distribution by the way the SPAD detector works,

$$
\operatorname{Pr}(k)= \begin{cases}1-p, & \text { if } k=0, \\ p, & \text { if } k=1,\end{cases}
$$

where

$$
p=\frac{\lambda}{1 !} e^{-\lambda}+\frac{\lambda^{2}}{2 !} e^{-\lambda}+\frac{\lambda^{3}}{3 !} e^{-\lambda}+\ldots
$$

One can calculate the Fano factor for the Bernoulli distribution and it turns out to be less than one,

$$
F=\frac{\operatorname{var}(\mathcal{N})}{\langle\mathcal{N}\rangle}=\frac{p(1-p)}{p}=1-p
$$

The value of $p$ for the data set from Fig. 5 was calculated to be $p=0.00330$. This value of $p$, when used in Eq. (7), perfectly explains the deviation of the Fano factor from one towards sub-Poissonian photostatistics. 


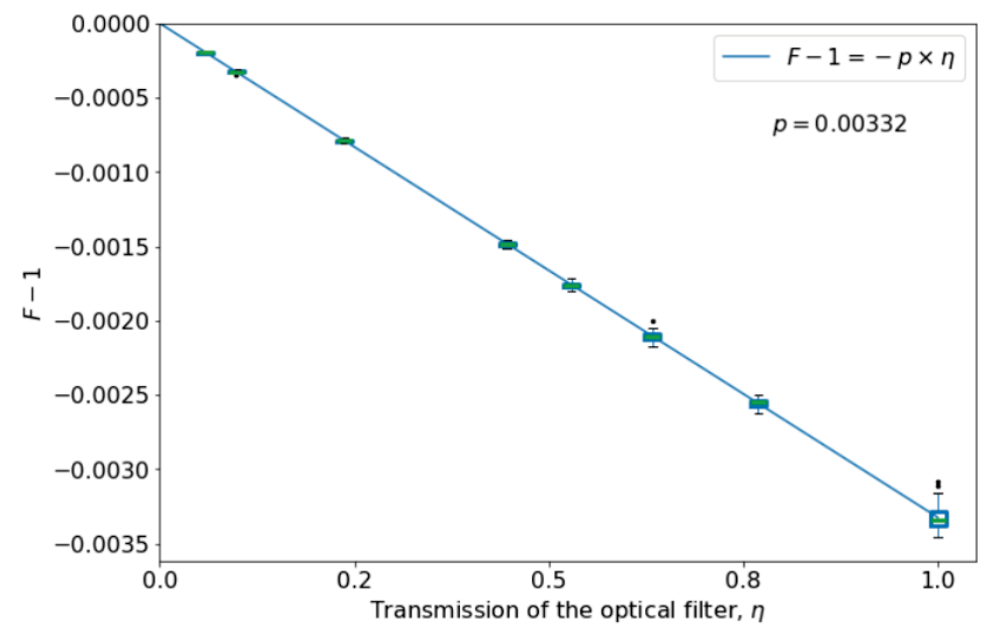

FIG. 7. The measured Fano factor as a function of the transmission of the optical neutral density filter in front of the SPAD detector. Also, linear interpolation for the measured data points.

We also decided to take measurements of the Fano factor with seven different neutral density filters in front of the SPAD detector and make a plot of the Fano factor as a function of the transmission of the filter. The result of these measurements are shown in Fig. 7.

The idea of this measurement was to verify the following relation between the intrinsic and the measured Fano factor,

$$
F_{\text {measured }}-1=\eta\left(F_{\text {intrinsic }}-1\right),
$$

where $\eta$ is the detection efficiency of the system. As can be seen in Fig. 7, the measured dependence of the Fano factor on the transmission of the neutral density filter follows a straight line indeed. It is noteworthy, that Eq. (8) shows that if the detection efficiency of the system is very low, then the right-hand side of Eq. (8) goes to zero, and any intrinsic photostatistics will look like Poissonian when measured. Therefore, it is crucial to have a high detection efficiency. This is exactly why we use the SPAD detector in our experiment, because it has the highest available quantum efficiency, namely, $65 \%$.

Although the measurement of the Fano factor was affected by the principle of operation of the SPAD detector, the distribution of interarrival times of the photon detection events should be the same for a binary detector and a detector able to resolve the number of detected photons. For both types of collected data, namely, Eq. (1) (binary detector) and Eq. (3) (detector with photon number resolution), the distribution of interarrival times (in units of IOTA revolutions) is geometric, i.e., 


$$
\operatorname{Pr}(k)=(1-p)^{k-1} p,
$$

where $k$ is the interarrival time in units of IOTA revolutions. One can see in Fig. 8 that the empirical distribution of interarrival times agrees well with the expected geometric distribution.

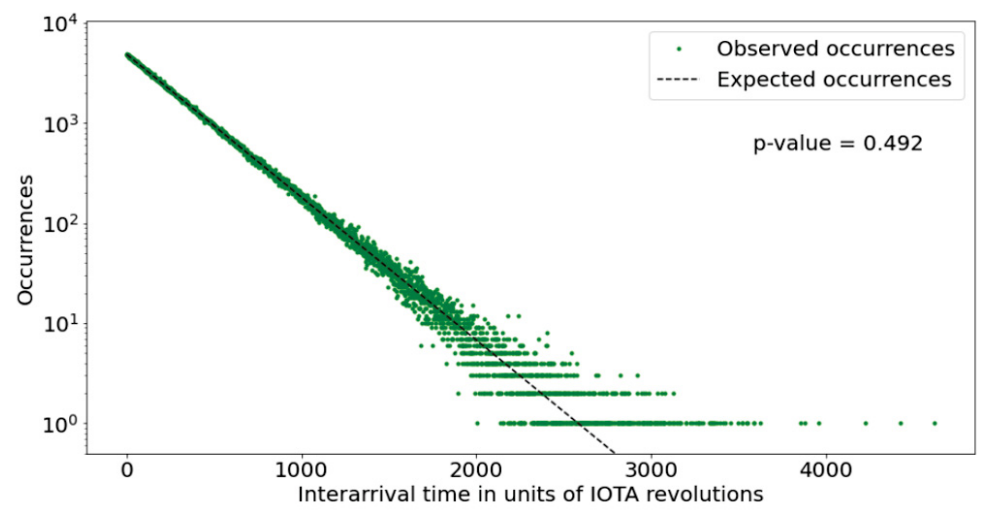

FIG. 8. Histogram of interarrival times of the photon detection events. Also, a fit with a geometric distribution.

To quantitatively describe the agreement, we used the Chi-square goodness-of-fit test. The $p$-value for the fit in Fig. 8 is 0.492 . Conventionally, the deviation is believed to be statistically insignificant if the $p$-value is greater than 0.05. Therefore, it is reasonable to say that in our experiment the distribution of interarrival times is geometric indeed.

We also studied the distribution of the number of photon counts in a certain time window, equal to 1000 IOTA revolutions in this specific example. The measured distribution is shown in Fig. 9.

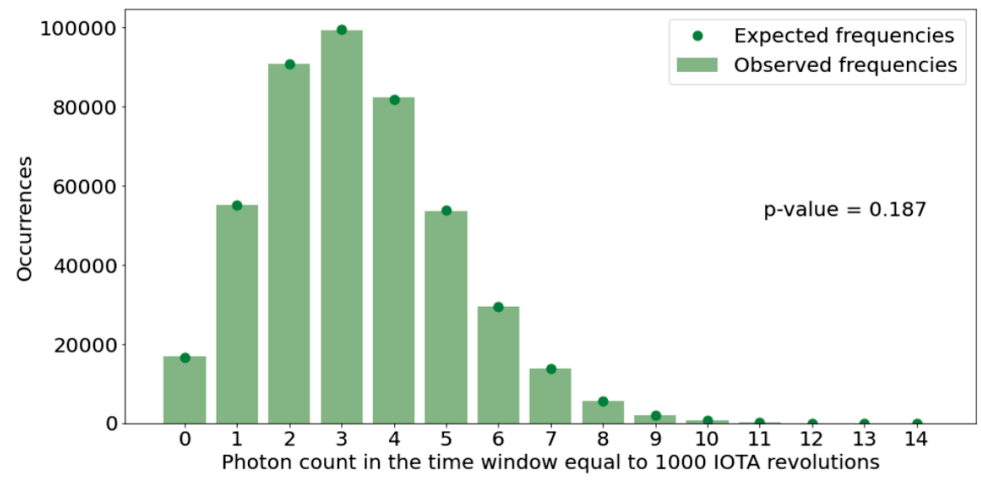

FIG. 9. Measured distribution of the number of detection events in a time window equal to 1000 IOTA revolutions. Also, a fit with binomial distribution. 
It is well known that the number of positive outcomes $k$ in a series of $n$ (in our example $n=1000$ ) Bernoulli trials follows the binomial distribution,

$$
\operatorname{Pr}(k)=\left(\begin{array}{l}
n \\
k
\end{array}\right) p^{k}(1-p)^{n-k}
$$

As we can see in Fig. 9, the measured occurrences actually follow the expected binomial distribution very well, which is also confirmed by the Chi-square goodness-of-fit test ( $p$-value $>0.05)$.

We also verified that the distribution of interarrival times is memoryless, which means that the probability to detect a photon in the future revolutions, does not depend on the past detection events. Quantitatively it can be expressed as

$$
\operatorname{Pr}(X>m+n \mid X \geq m)=\operatorname{Pr}(X>n),
$$

where $X$ is an interarrival time in units of IOTA revolutions. This property was also quantitatively confirmed using the Chi-square goodness-of-fit test. Summing up, all considered aspects of the collected data meet the criteria of a random memoryless Bernoulli process.

\section{POSSIBLE APPLICATIONS OF COLLECTED DATA}

As was mentioned before, for each detection, we also know the detection time relative to IOTA revolution marker. When we plotted this time as a function of IOTA revolution number for a single electron in IOTA, see Fig. 10, we observed a sinusoidal motion - this is the synchrotron motion of the single electron circulating in IOTA.

The vertical error bars represent the transit time spread of the SPAD detector, namely, 0.35 ns. The low rate of dark counts also adds a small contribution to the noise.

From these data (Fig. 10), we estimated the synchrotron motion period in IOTA: $T_{s}=2358 \pm$ 10 IOTA revolutions.

In Fig. 10, we studied the longitudinal motion of the single electron on a rather small time scale of a few milliseconds. On a larger time scale, the amplitude of the synchrotron motion is no longer constant because of quantum excitation, radiation damping, and, possibly, scattering in the background gas. To illustrate this we plotted RMS of the detection time relative to IOTA revolution marker in a time window of $0.1 \mathrm{~s}$ as a function of time for a single electron circulating in IOTA in Fig. 11. 


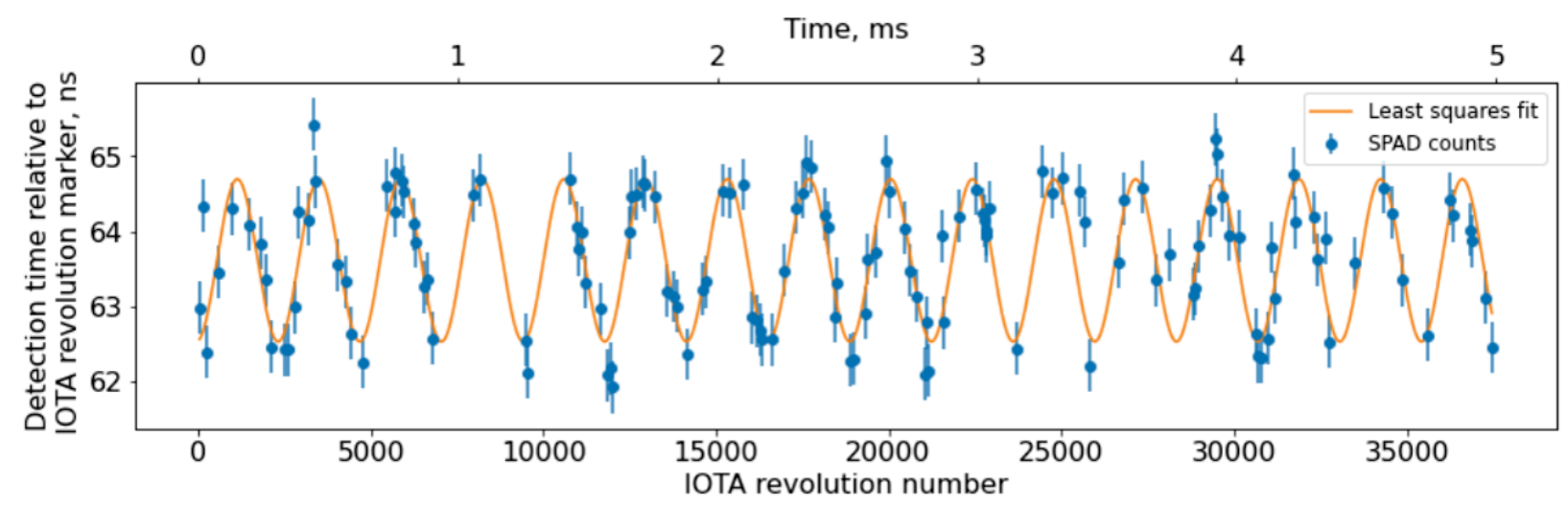

FIG. 10. Arrival time of a photocount relative to IOTA revolution marker as a function of IOTA revolution number for a single electron. A sinusoidal motion can be noticed - synchrotron motion of the single electron.

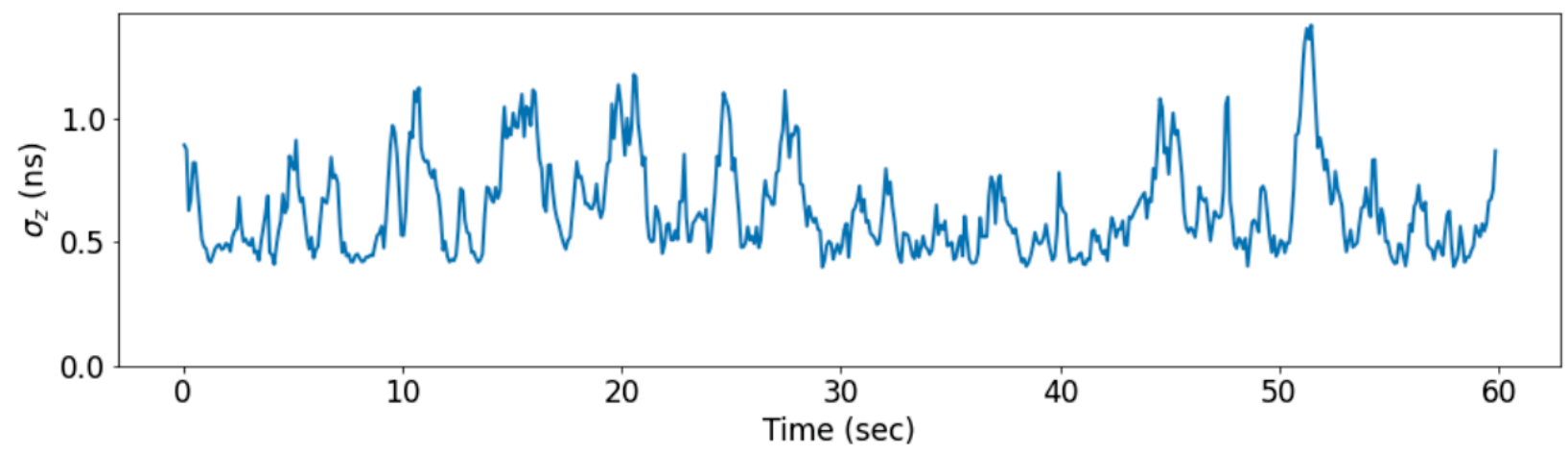

FIG. 11. RMS of the detection time relative to IOTA revolution marker in a time window of $0.1 \mathrm{~s}$ as a function of time for a single electron circulating in IOTA.

This plot can be understood as an attempt to illustrate the RMS of the longitudinal position of the electron in a time window of $0.1 \mathrm{~s}$ as a function of time. However, it is not strictly correct, because there is also a considerable contribution from the transit time spread of the SPAD detector, about $0.35 \mathrm{~ns}$ according to the specifications. Nonetheless, in Fig. 11, we can clearly see the constant fight between quantum excitation (when the RMS size increases) and radiation damping (when the RMS size decreases). Also, there might be some events of scattering in the background gas (presumably very rare). These data can potentially be used to calculate the radiation damping time in IOTA and study scattering in the background gas.

Of course, on the time scale of Fig. 11, not only the amplitude of synchrotron motion changes, but also the slow phase $\phi$, which enters the equation for synchrotron motion of the electron as

$$
z(t)=A_{z} \cos (\Omega t+\phi(t))
$$

where $A_{z}$ is the amplitude of synchrotron motion and $\Omega$ is the synchrotron motion angular fre- 
quency.

Below we attempt to find evolution of the slow phase $\phi$ in the same data set as in Fig. 11. On average there is about 8 detection events per one period of synchrotron motion. Therefore, we cannot detect change of $\phi$ on a scale of a few synchrotron motion periods. However, the collected data points are dense enough to flawlessly count the number of full synchrotron motion revolutions completed before a certain moment in time. This can be done by fitting the full 60 sec-long data in small pieces. Figure 12 shows three such pieces, about 20 synchrotron motion periods long, with 3 period long overlaps. The red and blue vertical lines represent the beginnings and the endings of the pieces, respectively. In the full, concatenated fit, in the regions of overlap, we linearly transition from one fit to the other, so that the final curve is smooth.

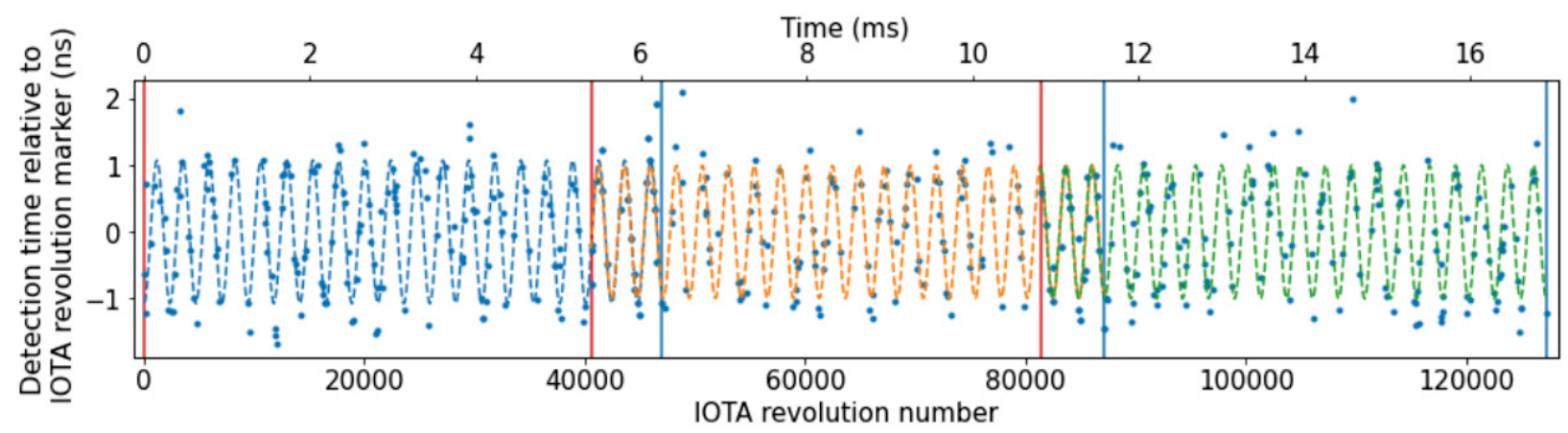

FIG. 12. Detection time relative to IOTA revolution marker (the average was subtracted) as a function of time. Piece-by-piece fitting of the full 60 sec-long data set (only three pieces are shown).

Using the full 60 sec-long fit, we can plot the number of synchrotron motion revolutions VS. time. We used the times of negative-to-positive intersections of the full fit curve with the time axis, however, one could also use, for example, times of the maximums of the fit curve, minimums of the fit curve, etc. Using these data and least squares linear regression we found a more accurate estimate (compared to the one above, based on Fig. 10) for the average synchrotron motion period, $2353.369 \pm 0.001$ IOTA revolutions. Then, after subtracting the regular component $\Omega t$ (based on the average synchrotron motion period), we were able to plot the slow phase $\phi$ as a function of time, see Fig. 13.

Figure 13 shows the effect of quantum excitation and radiation damping (and possibly scattering in the background gas) on the slow phase $\phi$ of synchrotron motion. Finally, since we have Fig. 11, which essentially represents the evolution of the amplitude of synchrotron motion, and Fig. 13, which represents the evolution of the slow phase of synchrotron motion, we can combine these results to plot the trajectory of the single electron in the longitudinal phase space, see Fig. 14. 


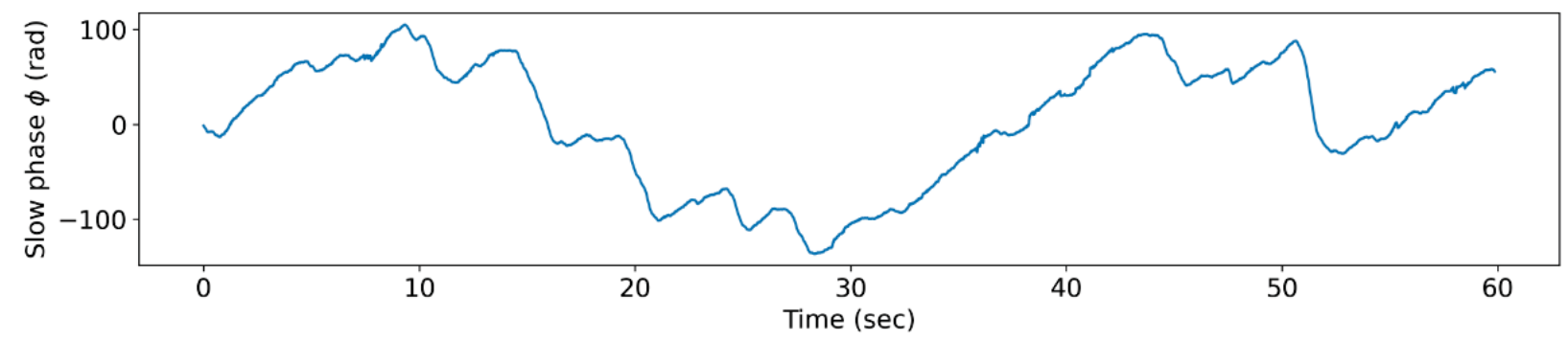

FIG. 13. Slow phase $\phi$ of synchrotron motion as a function of time.

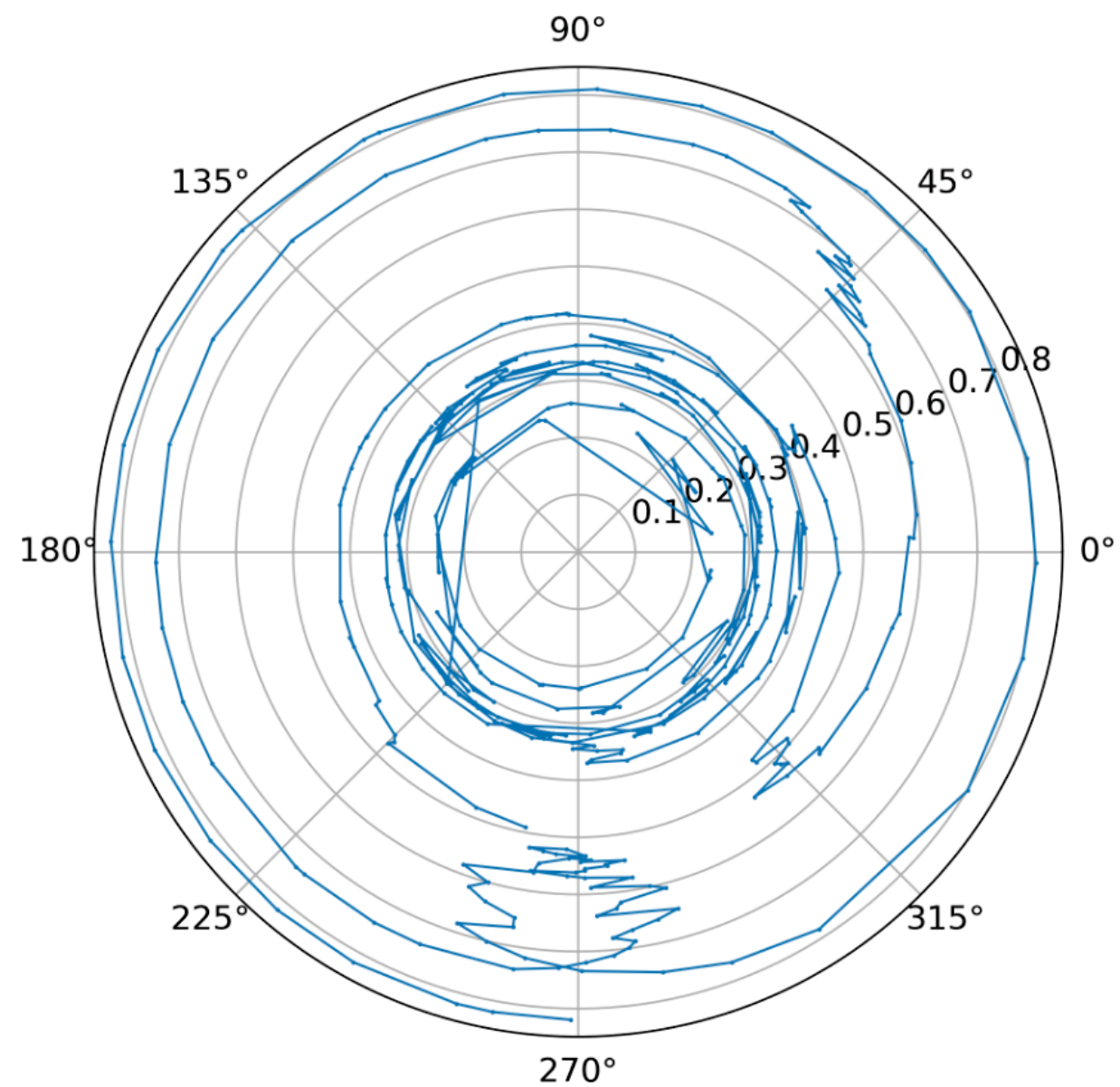

FIG. 14. Trajectory of the single electron in the longitudinal phase space during $2.5 \mathrm{sec}$ of motion. The radius represents $\sigma_{z}$ (ns) from Fig. 11 (but with subtracted in quadrature transit time spread of the SPAD - $0.35 \mathrm{~ns}$ ). The angle is the slow phase $\phi$ from Eq. (12).

We only show the first $2.5 \mathrm{sec}$ of the enitre $60 \mathrm{sec}$ long data set in Fig. 14 because the full trajectory would cover the whole circle. The trajectory reminds of Brownian motion (random walk), indeed. However, one can see that there is some predominant direction of rotation at large and small amplitudes, and truly random motion at average amplitudes — this is the sign of 
nonlinearity of the synchrotron oscillations in IOTA (rotation frequency depends on the amplitude). Similar effects were previously observed in [14].

\section{CONCLUSIONS}

For a single electron in a ring and a binary photon detector, we verified that photon emission is a random memoryless Bernoulli process, with no observable deviations. Using two or more binary detectors will allow us to measure the 2-photon and 3-photon rates. A single electron in a storage ring offers a unique probe to test our understanding of a charged particle's behavior in an accelerator, including the interaction with its own fields. This is a part of the program of the upcoming Optical Stochastic Cooling experiment in IOTA.

\section{FUNDING}

The project is funded by a Fermilab LDRD grant [15] and supported by SLAC and UChicago.

[1] I. Lobach, S. Nagaitsev, A. Valishev, A. Romanov, G. Stancari, A. Halavanau, and Z. Huang, Measurement of Spontaneous Undulator Radiation Statistics Generated by a Single Electron (URSSE), https://cdcvs.fnal.gov/redmine/attachments/57543/Lobach_URSSE_Proposal_ 2019-12-12_v1.pdf (2019).

[2] R. J. Glauber, Coherent and incoherent states of the radiation field, Phys. Rev. 131, 2766 (1963).

[3] R. J. Glauber, The quantum theory of optical coherence, Phys. Rev. 130, 2529 (1963).

[4] R. J. Glauber, Some notes on multiple-boson processes, Phys. Rev. 84, 395 (1951).

[5] T. Chen, Photon statistics of coherent harmonic radiation of a linac free electron laser, Ph.D. thesis, Duke University (1999).

[6] URSSE experiment website, https://cdcvs.fnal.gov/redmine/projects/fur/wiki/Wiki.

[7] O. Chubar, A. Fluerasu, L. Berman, K. Kaznatcheev, and L. Wiegart, Wavefront propagation simulations for beamlines and experiments with "Synchrotron Radiation Workshop", in J. Phys. Conf. Ser., Vol. 425 (IOP Publishing, 2013) p. 162001.

[8] I. Lobach, V. Lebedev, S. Nagaitsev, A. Romanov, G. Stancari, A. Valishev, A. Halavanau, Z. Huang, and K.-J. Kim, Statistical properties of spontaneous synchrotron radiation with arbitrary degree of coherence, submitted to PRAB (2020). 
[9] I. Lobach, V. Lebedev, S. Nagaitsev, A. Romanov, G. Stancari, A. Valishev, A. Halavanau, Z. Huang, and K.-J. Kim, Statistical properties of undulator radiation in the IOTA storage ring, arXiv preprint arXiv:1912.06737 (2019).

[10] M. C. Teich, T. Tanabe, T. C. Marshall, and J. Galayda, Statistical properties of wiggler and bendingmagnet radiation from the Brookhaven Vacuum-Ultraviolet electron storage ring, Phys. Rev. Lett. 65, 3393 (1990).

[11] F. Sannibale, G. Stupakov, M. Zolotorev, D. Filippetto, and L. Jägerhofer, Absolute bunch length measurements by incoherent radiation fluctuation analysis, Phys. Rev. ST Accel. Beams 12, 032801 (2009).

[12] V. Sajaev, Measurement of bunch length using spectral analysis of incoherent radiation fluctuations, in AIP Conf. Proc., Vol. 732 (AIP, 2004) pp. 73-87.

[13] T. Chen and J. M. Madey, Observation of sub-Poisson fluctuations in the intensity of the seventh coherent spontaneous harmonic emitted by a RF linac free-electron laser, Phys. Rev. Lett. 86, 5906 (2001).

[14] N. Vinokurov, Longitudinal motion in storage rings and quantum excitation, in Beam Measurement (World Scientific, 1999) pp. 108-123.

[15] Quantum effects in undulator radiation, Fermilab LDRD, L2019.025, June 2019 - September 2021, PI: Sergei Nagaitsev, Co-PIs: Ihar Lobach, Aleksandr Romanov, and Giulio Stancari. 